\title{
Caracterização de Hidrogéis Condutores Constituídos por PAAm e PEDOT/PSS por meio de Planejamento Fatorial
}

\author{
Fauze A. Aouada, Márcia R. de Moura, Emerson M. Girotto, Adley F. Rubira, Edvani C. Muniz \\ Departamento de Química, UEM
}

\author{
Gilsinei M. Campese \\ Departamento de Sistemas Químicos e Informática, UNICAMP
}

\begin{abstract}
Resumo: Neste trabalho, hidrogéis condutores do tipo semi-IPN constituídos por poliacrilamida (PAAm) e polímero intrinsecamente condutor poli(3,4-etileno dioxitiofeno)/poli(estireno-sulfonato) (PEDOT/PSS) foram sintetizados e suas propriedades hidrofílicas, mecânicas, condutoras e ópticas foram caracterizadas por meio de planejamento fatorial completo $2^{3}$ com ponto central. Para isso, utilizaram-se três fatores: concentrações de acrilamida (AAm) e N,N'-metileno-bis-acrilamida (MBAAm), e quantidade de PEDOT/PSS, em três níveis, sendo um deles o ponto central. O planejamento foi construído para avaliar os efeitos desses fatores (efeitos principais e efeitos de interação entre os fatores) nas seguintes propriedades dos hidrogéis estudados (respostas): grau de intumescimento, tensão máxima de compressão, condutância iônica e intensidade relativa de luz transmitida através dos hidrogéis estudados. Os resultados demonstraram que a estabilidade mecânica dos hidrogéis semi-IPN é preservada e a presença de PEDOT/PSS aumenta o entrelaçamento na matriz de PAAm. Observou-se também que as propriedades ópticas são fortemente influenciadas com a presença de PEDOT/PSS. Dessa forma, torna-se possível obter hidrogéis com hidrofilicidade, estabilidade mecânica e transparência controladas, o que é importante para a aplicação desses materiais, principalmente como dispositivos ópticos.
\end{abstract}

Palavras-chave: Hidrogéis condutores, planejamento fatorial, propriedades ópticas, estabilidade mecânica.

\section{Characterization of Conducting Hydrogels made with PAAm and PEDOT/PSS using Factorial Design}

\begin{abstract}
Conductive semi-IPN hydrogels made with polyacrylamide (PAAm) and the conductive polymer poly(3,4-ethylene dioxythiophene)/poly(styrenesulfonate) (PEDOT/PSS) were synthesized and the hydrophilicity, mechanical, ionic conductivity and optical properties were characterized through the $2^{3}$ factorial design. The experiments concerning the factorial design were randomly performed. The inputs were acrylamide (AAm), N,N'-methylene-bis-acrylamide (MBAAm) and PEDOT/PSS contents, each fixed in three levels, one being the central point. The outputs were the swelling degree, maximum compression stress, ionic conductance and relative intensity of light transmitted through the hydrogel. ANOVA was obtained using Statistical Software ${ }^{\circledR}$. The results of the twelve runs from the factorial design (eight runs from $2^{3}$ plus four central point replicates) were applied to evaluate the main effects and their interaction, in addition to decide if they are statistically significant. The results demonstrated that mechanical stability of semi-IPN hydrogels is preserved, with PEDOT/PSS creating additional entanglements within the PAAm network. In addition, the optical properties are strongly influenced by the presence of PEDOT/PSS. In summary, hydrogels could be synthesized with controlled hydrophilicity, transparency and mechanical stability, which is important for optical devices.
\end{abstract}

Keywords: Conducting hydrogels, factorial design, optic properties, mechanical stability.

\section{Introdução}

O planejamento fatorial tem sido muito aplicado em pesquisas básicas e tecnológicas e é classificado como um método multivariado, onde muitos fatores podem ser variados ao mesmo tempo ${ }^{[1,2]}$. As análises de tabelas de variância (ANOVA) são utilizadas para determinar quais fatores (e seus efeitos) são estatisticamente significativos. A análise da significância dos efeitos pode ser realizada também através dos valores de teste $\mathrm{F}$, que pode ser obtido por meio da razão entre as médias quadráticas associada à regressão e associada aos resíduos ${ }^{[3]}$. Os valores calculados de $\mathrm{F}$ são comparados com valor crítico $\mathrm{Fv}_{1} \mathrm{v}_{2}$ (ou $\mathrm{F}_{0}$, fornecido por tabelas estatísticas) no limite de confiança de $95 \%$, para $v_{1}$ e $v_{2}$ graus de liberdade. Uma grande diferença entre $F$ e $F_{0}$ $\left(\mathrm{F}>\mathrm{F}_{0}\right)$ mostra que o efeito analisado é estatisticamente significativo ${ }^{[3,4]}$. Uma outra ferramenta que pode ser utilizada nos estudos dos efeitos é a Metodologia de Superfície de Respostas (MSR) $)^{[5,6]}$. Essa analisa os efeitos e suas interações através de modelos empíricos.

A utilização do ponto central ${ }^{[7]}$ permite adicionar um terceiro nível para cada fator, possibilitando assim a realização

Autor para correspondência: Edvani C. Muniz, Departamento de Química, Universidade Estadual de Maringá, CEP: 87020-900, Maringá, PR, Brasil. 
do estudo do fatorial através da Metodologia de Superfície de Respostas (MSR), bem como quantificar a significância de possível curvatura e erros associados aos efeitos individuais e de interações ${ }^{[3]}$.

Hidrogéis são materiais formados por redes de polímeros hidrofílicos que possuem habilidade de absorver grande quantidade de água ${ }^{[8,9]}$. O confinamento de materiais eletroópticos (p. exemplo, polímeros condutores) em matrizes poliméricas tem despertado um especial interesse devido às suas aplicações tecnológicas, dentre estas as mais conhecidas são as "cortinas eletrônicas". Outra forma de confinamento de materiais eletro-ópticos consiste em introduzi-los no interior de hidrogéis, os quais passam a apresentar diferentes propriedades ${ }^{[10]} \mathrm{em}$ relação aos componentes puros.

Neste trabalho os hidrogéis semi-IPN foram caracterizados através de um planejamento fatorial completo $2^{3}$ com ponto central. Para efetuar as análises dos dados utilizou-se o programa computacional Statistical Software ${ }^{\circledR}$ (versão 6.0). Os resultados do planejamento fatorial podem ser expressos por meio da equação (modelo matemático):

$$
\begin{gathered}
\mathrm{y}=\mathrm{b}_{0}+\mathrm{b}_{\mathrm{A}} \mathrm{A}+\mathrm{b}_{\mathrm{B}} \mathrm{B}+\mathrm{b}_{\mathrm{C}} \mathrm{C}+\mathrm{b}_{\mathrm{AB}} \mathrm{A} * \mathrm{~B}+ \\
\mathrm{b}_{\mathrm{AC}} \mathrm{A} * \mathrm{C}+\mathrm{b}_{\mathrm{BC}} \mathrm{B} * \mathrm{C}+\mathrm{b}_{\mathrm{ABC}} \mathrm{A}^{* B} \mathrm{~B} \mathrm{C}^{-}
\end{gathered}
$$

onde y é a resposta, $\mathrm{b}_{0}$ é a média dos efeitos na resposta y, $b_{i}$ são os efeitos principais, $b_{i j k}$ são os efeitos de interação entre os fatores $\mathrm{A}, \mathrm{B}$ e $\mathrm{C}^{[11]}$. No presente trabalho, o fator $\mathrm{A}$ é a concentração molar de acrilamida (AAm), o fator B é a concentração de MBAAm (\% molar em relação a AAm) e o fator $\mathrm{C}$ é a quantidade de PEDOT/PSS (\% v:v). O objetivo principal deste estudo é avaliar, por meio da construção de planejamento fatorial, a influência desses fatores bem como suas interações sobre as propriedades (respostas) dos hidrogéis estudados: grau de intumescimento $(\mathrm{Q})$, tensão máxima de compressão $\left(\sigma_{\max }\right)$, condutância iônica (Cond. Ion.) e intensidade relativa de luz transmitida $\left(\mathrm{I} / \mathrm{I}_{0}\right)$.

\section{Experimental}

\section{Sintese dos hidrogéis semi-IPN constituídos por PAAm e PEDOT/PSS}

Os hidrogéis semi-IPN foram obtidos através de polimerização química do monômero AAm em solução aquosa contendo PEDOT/PSS e MBAAm. A concentração de TEMED, utilizado como catalisador, foi mantida constante em 2,85 $\mu \mathrm{mol} \mathrm{mL}-1$. Persulfato de sódio $\left(4,20 \mu \mathrm{mol} \mathrm{mL}{ }^{-1}\right)$ foi utilizado como iniciador da reação de polimerização via radical livre. Depois da mistura dos reagentes, a solução foi purgada borbulhando $\mathrm{N}_{2}$ durante 20 minutos. A solução resultante foi inserida entre duas placas de vidro $(12 \times 12 \mathrm{~cm})$ separadas por um espaçador de borracha $(3,0 \mathrm{~mm})$. Para a identificação das diferentes membranas poliméricas sintetizadas neste trabalho foi adotada a notação (A-M-P), onde A é a concentração molar de AAm, M é a concentração de
MBAAm (\% molar em relação a AAm) e P é a quantidade de PEDOT/PSS (em \% v:v).

\section{Grau de intumescimento (Q)}

Os hidrogéis foram imersos em água destilada e deixados intumescer até equilíbrio, a $25,0^{\circ} \mathrm{C}$. O grau de intumescimento $(\mathrm{Q})$ foi determinado através da razão entre a massa do hidrogel intumescido até equilíbrio e a massa do hidrogel seco.

\section{Tensão máxima de compressão $\left(\sigma_{\max }\right)$}

A resistência à compressão (rigidez) dos hidrogéis foi determinada por meio de testes de tensão máxima de compressão $\left(\sigma_{\max }\right)$, a $25,0{ }^{\circ} \mathrm{C}$. Foi utilizado o equipamento Texture Analyzer (Stevens L.F.R.A) com corpo de prova circular de $12,7 \mathrm{~mm}$ de diâmetro e velocidade de descida igual a $0,2 \mathrm{~mm} \mathrm{~s}^{-1}$. Os valores de $\sigma_{\max }$ foram obtidos através da razão entre a força necessária para comprimir em $1 \mathrm{~mm}$ as membranas dos hidrogéis e a área do corpo de prova.

\section{Intensidade relativa de luz transmitida (I/Io)}

Os hidrogéis (intumescidos até equilíbrio) foram cortados em forma circular e imersos em uma cela de vidro transparente contendo água destilada. A amostra foi colocada no interior de uma caixa fechada, protegida de luz externa, à temperatura constante de $25,0^{\circ} \mathrm{C}$. Um feixe proveniente de um laser $\mathrm{He}-\mathrm{Ne}(632,8 \mathrm{~nm})$ foi incidido na amostra e a quantidade de luz transmitida através da amostra foi medida por meio de um fotodiodo (EGG Optoeletronic modelo HUV 200 B), usado como detector, acoplado a um multímetro. Foi determinada a quantidade $\mathrm{I} / \mathrm{I}_{\mathrm{o}}$ sendo I a intensidade de luz através da amostra e $\mathrm{I}_{\mathrm{o}}$ a intensidade de luz que chega no detector na ausência de amostra.

\section{Condutância iônica (cond. Ion.)}

A condutância iônica foi obtida por meio de medidas de resistividade iônica apresentada pelo hidrogel inserido entre dois eletrodos circulares (área de $706 \mathrm{~mm}^{2}$ ) construídos em aço inox, usando o método de duas pontas. A condutância iônica é definida como sendo inverso da resistividade iônica ( $\rho)$ sendo que $\rho$ é calculada por meio da equação abaixo ${ }^{[12]}$.

$$
\rho=\frac{\frac{(d)^{2} \pi}{4}}{w} \frac{V}{i}
$$

onde d é o diâmetro do eletrodo, w é a espessura do hidrogel, V é o potencial aplicado e i é a corrente gerada.

\section{Resultados e Discussão}

Nos planejamentos experimentais onde os fatores são analisados em 3 níveis, é comum codificá-los usando (+) para o nível maior, (0) para o ponto central e (-) para o nível menor. A atribuição dos valores para os níveis superior, central e inferior é feita de forma arbitrária e não interfere na reali- 
zação dos experimentos ou na interpretação dos resultados, além de permitir que o planejamento seja esquematizado na forma de matrizes ${ }^{[3]}$.

Na Tabela 1 são apresentados os valores reais dos níveis superior (+) e inferior (-) e para o ponto central (0) para cada um dos fatores estudados. Na Tabela 2 é apresentada a matriz planejamento com os valores obtidos nos diferentes experimentos para as quatro diferentes respostas.

Os resultados obtidos do planejamento para a resposta grau de intumescimento $(\mathrm{Q})$, considerando a ANOVA (Tabela 3), demonstraram que apenas os efeitos dos fatores A e B são significativos. Os efeitos de interação de dois ou mais fatores são todos não significativos. Para o grau de intumescimento, o modelo obtido foi:

Tabela 1. Fatores e níveis utilizados no planejamento fatorial.

\begin{tabular}{cccc}
\hline Fatores & $\begin{array}{c}\text { Nível inferior } \\
(-)\end{array}$ & $\begin{array}{c}\text { Ponto central } \\
(\mathbf{0})\end{array}$ & $\begin{array}{c}\text { Nível superior } \\
(+)\end{array}$ \\
\hline A & 2,0 & 3,5 & 5,0 \\
B & 1,0 & 1,75 & 2,5 \\
C & 1,0 & 3,0 & 5,0 \\
\hline
\end{tabular}

$\mathrm{A}=\mathrm{AAm}\left(\mathrm{mol} \mathrm{L}{ }^{-1}\right), \mathrm{B}=\operatorname{MBAAm}(\%$ molar em relação a AAm $)$ e $\mathrm{C}=\mathrm{PEDOT} / \mathrm{PSS}(\% \mathrm{v}: \mathrm{v})$.

$$
\mathrm{Q}=6,79-1,18 \mathrm{~A}-1,74 \mathrm{~B}
$$

indicando que o fator B é mais importante que o fator $\mathrm{A}$. Na Figura 1 é mostrada a superfície de resposta obtida para o grau de intumescimento em função dos fatores A e B. A curvatura da superfície de resposta demonstrou ser significativa para os intervalos estudados. Assim, um modelo quadrático, ao invés do modelo linear (Equação 3) pode ser mais adequado para explicar o efeito desses fatores no grau de intumescimento ${ }^{[3]}$.

Pode-se observar ainda que os fatores A e B contribuem negativamente para os valores de Q. Assim, mantendo A ou B em seus níveis superiores, possibilita o aumento na densidade de cadeias poliméricas e no grau de reticulação dos hidrogéis, contribuindo para redução da quantidade de água adsorvida pelos hidrogéis. Por meio da superfície de resposta pode-se perceber que maiores valores de $\mathrm{Q}$ são obtidos para os níveis inferiores dos fatores A e B.

Na Tabela 4 são mostrados os dados de ANOVA para a tensão máxima de compressão $\left(\sigma_{\max }\right)$ obtidos para os hidrogéis semi-IPN contendo PEDOT/PSS confinado.

Tabela 2. Matriz planejamento e valores das respostas para cada experimento realizado.

\begin{tabular}{|c|c|c|c|c|c|c|c|}
\hline Experimentos & $\mathbf{A}$ & B & $\mathbf{C}$ & $Q(g / g)$ & $\sigma_{\max }(\mathbf{k P a})$ & $\mathbf{I} / \mathbf{I}_{0}$ & Cond. Ion. $\left(\mu \mathrm{S} \mathrm{cm}^{-1}\right)$ \\
\hline 1 & - & - & - & 9,93 & 21,68 & 0,834 & 17,40 \\
\hline 2 & + & - & - & 6,81 & 64,94 & 0,937 & 3,61 \\
\hline 3 & - & + & - & 5,86 & 38,30 & 0,964 & 11,74 \\
\hline 4 & + & + & - & 4,13 & 76,32 & 0,954 & 3,21 \\
\hline 5 & - & - & + & 10,12 & 19,24 & 0,273 & 16,50 \\
\hline 6 & + & - & + & 7,27 & 65,85 & 0,349 & 3,83 \\
\hline 7 & - & + & + & 5,97 & 39,94 & 0,278 & 11,26 \\
\hline 8 & + & + & + & 4,22 & 76,37 & 0,530 & 3,56 \\
\hline 9 & 0 & 0 & 0 & 5,48 & 74,33 & 0,551 & 3,49 \\
\hline 10 & 0 & 0 & 0 & 5,55 & 73,24 & 0,600 & 3,73 \\
\hline 11 & 0 & 0 & 0 & 5,51 & 77,66 & 0,529 & 3,56 \\
\hline 12 & 0 & 0 & 0 & 5,55 & 76,53 & 0,543 & 3,90 \\
\hline
\end{tabular}

Tabela 3. Análise de variância (ANOVA) para os fatores A, B e C e suas interações na resposta grau de intumescimento (Q).

\begin{tabular}{lccccc}
\hline \multicolumn{1}{c}{ Fonte } & Soma quadrática & Grau de liberdade & Média quadrática & teste-F & P \\
\hline \multicolumn{1}{c}{ Modelo } & $\mathbf{3 5 , 4 9}$ & $\mathbf{2}$ & $\mathbf{1 7 , 7 4}$ & $\mathbf{1 5 5 , 6 1}$ & $<\mathbf{0 , 0 0 0 1}$ \\
\hline A & 11,16 & 1 & 11,16 & 97,89 & $<0,0001$ \\
B & 24,33 & 1 & 24,33 & 213,42 & $<0,0001$ \\
C & 0,082 & 1 & 0,082 & 0,72 & 0,1090 \\
A B & 0,800 & 1 & 0,800 & 7,02 & 0,0359 \\
A C & 0,011 & 1 & 0,011 & 0,0967 & 0,2863 \\
B C & 0,021 & 1 & 0,021 & 0,18 & 0,2095 \\
A B C & 0,014 & 1 & 0,014 & 0,12 & 0,2554 \\
Curvatura & $\mathbf{4 , 2 8}$ & $\mathbf{1}$ & $\mathbf{4 , 2 9}$ & $\mathbf{3 7 , 4 9}$ & $<\mathbf{0 , 0 0 0 1}$ \\
Residual & 0,91 & 8 & 0,114 & - & - \\
Falta de ajuste & 0,91 & 5 & 0,182 & 156,89 & 0,0008 \\
Erro puro & $3,48 \times 10^{-3}$ & 3 & $1,16 \times 10^{-3}$ & - & - \\
Total & 40,68 & 11 & - & -
\end{tabular}




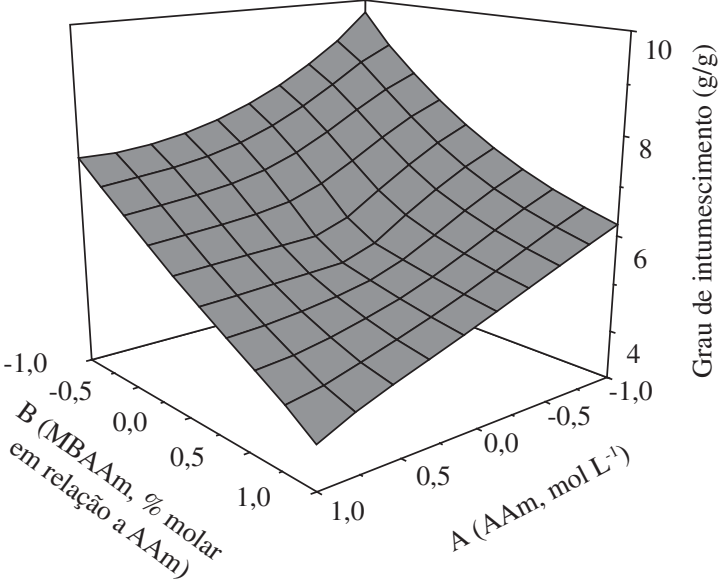

Figura 1. Superfície de resposta obtida para grau de intumescimento (Q) em função dos fatores A e B.

Por meio da análise dos dados da Tabela 4 observa-se que os fatores A e B também são estatisticamente significativos para $\sigma_{\max }$. O modelo obtido para o intervalo estudado foi:

$\sigma_{\text {max }}=50,33+20,54 \mathrm{~A}+7,40 \mathrm{~B}-1,93 \mathrm{~A} * \mathrm{~B}$

Ao contrário de $\mathrm{Q}$, os fatores A e B contribuem positivamente para a resposta $\sigma_{\max }$. Assim, a manutenção dos fatores A e B em seus níveis superiores reflete em maiores valores de $\sigma_{\max }$. Observa-se também que o fator A é mais importante que B. Dessa forma, dobrando os valores para esses dois fatores proporcionará aumento de quase 3 vezes nessa resposta quando comparada ao efeito do fator B. Este efeito pode ser visualizado por meio da superfície de resposta apresentada na Figura 2.

Alteração no nível do fator C (do nível inferior para o nível superior, ou vice-versa) não proporciona mudança significativa nos valores de Q e de $\sigma_{\max }$. É conhecido que em hidrogéis do tipo semi-IPN a presença de cadeias de um segundo polímero (não reticulado) pode ocasionar um aumento na compactação dos hidrogéis ${ }^{[13]}$. No entanto, pela análise dos dados da Tabela 4 tal compactação não ocorreu quando a concentração de PEDOT/PSS nos hidrogéis, fator C, passa de

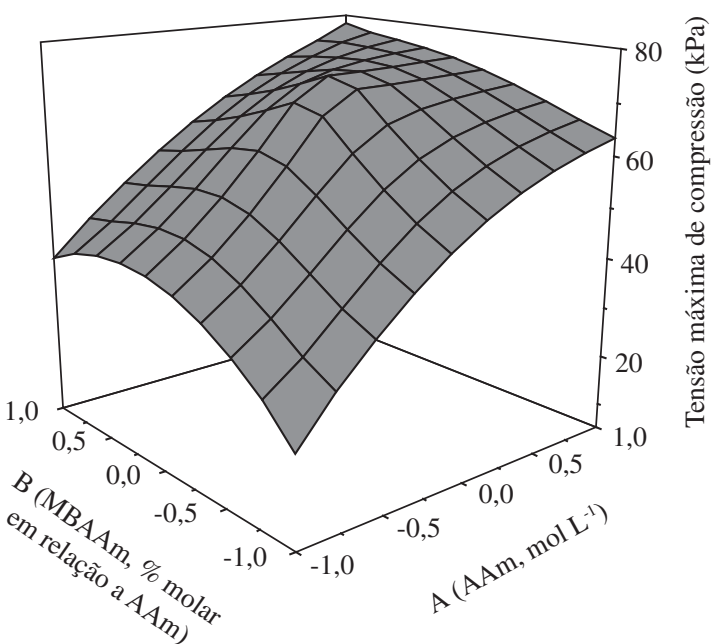

Figura 2. Superfície de resposta obtida para $\sigma_{\max }$ em função dos fatores A e B.

1,0\% (nível inferior) para 5,0\% (nível superior). Finalmente, o efeito de interação entre os fatores A e B é negativa, isto é, aumentado-se simultaneamente os níveis dos fatores A e B haverá um pequena redução na $\sigma_{\max }$. Isto é um exemplo de efeito de interação antagônico, onde a variação dos fatores, individualmente, do nível inferior para superior aumenta o valor da resposta, ao passo que uma variação simultânea dos fatores A e B provoca redução na resposta.

Aouada e colaboradores ${ }^{[14]}$, ao investigarem diversas propriedades de hidrogéis com PEDOT/PSS confinado, similares aos desenvolvidos neste trabalho, demonstraram que os valores do módulo de elasticidade, $E$, nos hidrogéis semi-IPN são maiores quando comparados aos hidrogéis de PAAm sem PEDOT/PSS. Por exemplo, para o sistema (2-1-P), os valores de $E(\mathrm{kPa})$ foram $27,02 \pm 1,38 ; 34,18 \pm 1,27$ e $33,03 \pm 0,50$ para $\mathrm{P}$ igual a $0 ; 1,0$ e $5,0 \%$, respectivamente. Isso mostra que a concentração de PEDOT/PSS de 1,0\% (v:v) é suficiente para causar a mesma compactação observada nos respectivos hidrogéis contendo 5,0\% (v:v) desse polímero.

Tabela 4. Análise dos fatores A, B e C e suas interações na resposta tensão máxima de compressão $\left(\sigma_{\max }\right)$.

\begin{tabular}{lccccc}
\hline \multicolumn{1}{c}{ Fonte } & Soma quadrática & Grau de liberdade & Média quadrática & teste-F & P \\
\hline \multicolumn{1}{c}{ Modelo } & $\mathbf{3 8 4 3 , 2 3}$ & $\mathbf{3}$ & $\mathbf{1 2 8 1 , 0 8}$ & $\mathbf{5 2 9 , 3 7}$ & $<\mathbf{0 , 0 0 0 1}$ \\
\hline A & 3375,13 & 1 & 3375,13 & 1394,68 & $<0,0001$ \\
B & 438,38 & 1 & 438,38 & 181,14 & $<0,0001$ \\
C & 0,0030 & 1 & 0,0030 & $1,24 \times 10^{-3}$ & 0,9794 \\
A B & 29,72 & 1 & 29,72 & 12,29 & 0,0099 \\
A C & 0,39 & 1 & 0,39 & 0,016 & 0,07777 \\
B C & 1,30 & 1 & 1,30 & 0,32 & 0,6117 \\
A B C & 3,05 & 1 & 3,05 & 1,26 & 0,4499 \\
Curvatura & $\mathbf{1 6 8 1 , 3 7}$ & $\mathbf{1}$ & $\mathbf{1 6 8 1 , 3 7}$ & $\mathbf{6 9 4 , 7 8}$ & $<\mathbf{0 , 0 0 0 1}$ \\
Residual & 16,93 & 7 & 2,42 & - & - \\
Falta de ajuste & 4,74 & 4 & 1,18 & 0,29 & 0,8677 \\
Erro puro & 12,19 & 3 & 4,06 & - & - \\
Total & 5541,52 & 11 & - & - & -
\end{tabular}


Analisando a Tabela 5, referente aos dados de ANOVA para a resposta $\mathrm{I} / \mathrm{I}_{0}$ observa-se que o fator $\mathrm{C}$ é a única variável significativa. Assim, obteve-se o seguinte modelo:

$$
\mathrm{I} / \mathrm{Io}=0,64-0,28 \mathrm{C}
$$

Observa-se que o fator $\mathrm{C}$, fator estatisticamente significativo, contribui negativamente na resposta. $\mathrm{O}$ aumento da quantidade de PEDOT/PSS no interior dos hidrogéis de PAAm conduz a redução expressiva nos valores de $\mathrm{I} / \mathrm{I}_{0}$. Isto ocorre devido à forte coloração que passa a predominar no material e que é característica do PEDOT/PSS. A respectiva superfície de resposta, apresentada na Figura 3, apresenta uma leve curvatura em relação ao fator $\mathrm{A}$, mas que, pelos dados da ANOVA, não é estatisticamente significativa.

Analisando os dados constantes na Tabela 6, referentes à ANOVA para a resposta condutância iônica (Cond. Ion.) observa-se que os fatores A e B contribuem forte e negativamente para essa resposta. Assim, menores valores para qualquer desses fatores provoca redução na condutância iônica do hidrogel. No entanto, se o fator C for mantido na faixa de concentração estudada (entre 1,0-5,0\%) não será significativo para a condutância iônica do hidrogel de PAAm-PEDOT/PSS.

O modelo obtido para condutância iônica foi:

Cond. Ion. $=8,89-5,34 \mathrm{~A}-1,45 \mathrm{~B}+1,28 \mathrm{~A} * \mathrm{~B}$

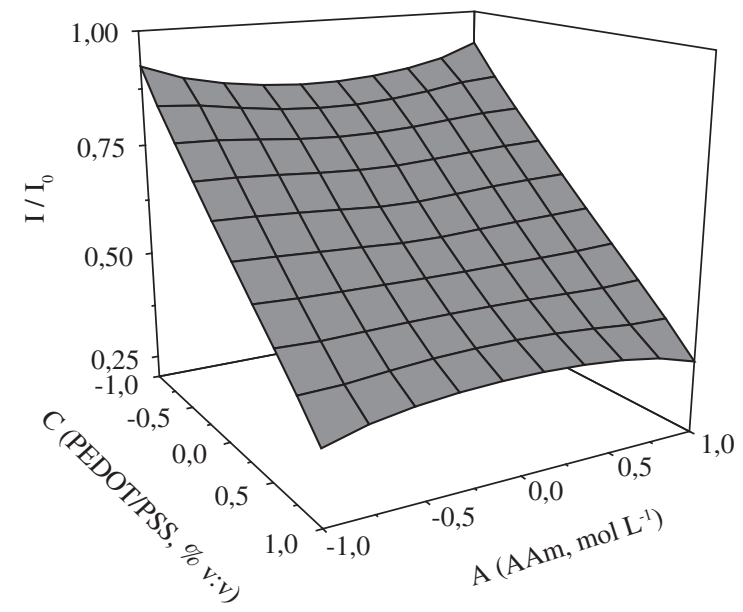

Figura 3. Superfície de resposta obtida para $I / I_{0}$ em função dos fatores A e C.

Tabela 5. Análise dos fatores A, B e C e suas interações na resposta intensidade relativa de luz transmitida (I/I $)$.

\begin{tabular}{lccccc}
\hline \multicolumn{1}{c}{ Fonte } & Soma quadrática & Grau de liberdade & Média quadrática & teste-F & P \\
\hline \multicolumn{1}{c}{ Modelo } & $\mathbf{0 , 6 4 1}$ & $\mathbf{1}$ & $\mathbf{0 , 6 4 1}$ & $\mathbf{1 0 1 , 2 6}$ & $<\mathbf{0 , 0 0 0 1}$ \\
\hline A & 0,0227 & 1 & 0,0227 & 18,89 & 0,1439 \\
B & 0,0143 & 1 & 0,0143 & 3,59 & 0,1797 \\
C & 0,641 & 1 & 0,641 & 101,26 & $<0,0001$ \\
A B & $5,8 \times 10^{-3}$ & 1 & 0,00058 & 0,092 & 0,6138 \\
A C & $6,61 \times 10^{-3}$ & 1 & 0,00661 & 1,04 & 0,2564 \\
B C & $1,45 \times 10^{-4}$ & 1 & 0,000145 & 0,023 & 0,7874 \\
A B C & 0,0101 & 1 & 0,01008 & 1,5924 & 0,2115 \\
Curvatura & $\mathbf{0 , 0 1 9}$ & $\mathbf{1}$ & $\mathbf{0 , 0 1 9}$ & $\mathbf{3 , 0 0}$ & $\mathbf{0 , 0 1 1 8 1}$ \\
Residual & 0,057 & 9 & $6,33 \times 10^{-3}$ & - & - \\
Falta de ajuste & 0,054 & 6 & $9,00 \times 10^{-3}$ & 9,44 & 0,0463 \\
Erro puro & $2,86 \times 10^{-3}$ & 3 & $9,53 \times 10^{-4}$ & - & - \\
Total & 0,71 & 11 & - & - & -
\end{tabular}

Tabela 6. Análise dos fatores A, B e C na resposta condutância iônica, Cond. Ion.

\begin{tabular}{lccccc}
\hline \multicolumn{1}{c}{ Fonte } & Soma quadrática & Grau de liberdade & Média quadrática & teste-F & P \\
\hline \multicolumn{1}{c}{ Modelo } & $\mathbf{2 5 7 , 6 2}$ & $\mathbf{3}$ & $\mathbf{8 5 , 8 7}$ & $\mathbf{8 5 8 , 7}$ & $<\mathbf{0 , 0 0 0 1}$ \\
\hline A & 227,80 & 1 & 227,80 & 2278 & $<0,0001$ \\
B & 16,73 & 1 & 16,73 & 167,3 & $<0,0001$ \\
C & 0,082 & 1 & 0,082 & 0,82 & 0,3406 \\
A B & 13,08 & 1 & 13,08 & 130,8 & $<0,0001$ \\
A C & 0,48 & 1 & 0,480 & 4,80 & 0,1537 \\
B C & 0,038 & 1 & 0,038 & 0,38 & 0,4568 \\
A B C & 0,011 & 1 & 0,011 & 0,11 & 0,6540 \\
Curvatura & $\mathbf{7 2 , 6 3}$ & $\mathbf{1}$ & $\mathbf{7 2 , 6 3}$ & $\mathbf{7 2 6 , 3}$ & $<0$ \\
Residual & 0,71 & 7 & 0,10 & - & - \\
Falta de ajuste & 0,61 & 4 & 0,15 & 4,41 & $-0,1235$ \\
Erro puro & 0,10 & 3 & 0,034 & - & - \\
Total & 330,95 & 11 & - & -
\end{tabular}




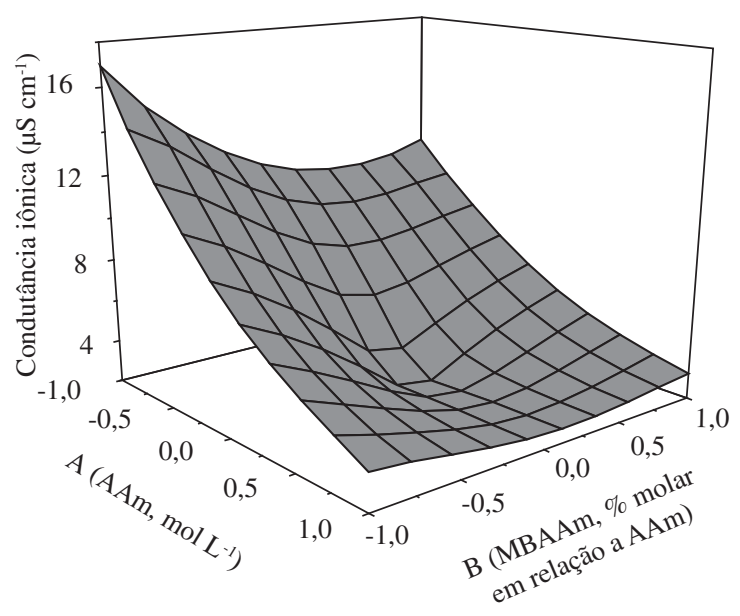

Figura 4. Superfície de resposta para Cond. Ion. em função dos fatores A e B.

A superfície de resposta, obtida para a resposta condutância iônica é mostrada na Figura 4. A curvatura apresentada é significativa e há evidente interação entre os fatores A e B. Assim, conclui-se que a condutividade iônica do hidrogel, nos níveis de PEDOT/PSS estudados, está relacionada à quantidade de água presente no hidrogel (o que está indiretamente ligado à concentração de AAm e de MBAAm, fatores $\mathrm{A}$ e $\mathrm{B}$, e não à quantidade de cadeias de PEDOT/PSS presentes no interior do hidrogel). Pode ser inferido que se a concentração do PEDOT/PSS for menor que $1,0 \%$ ou maior que $5,0 \%$ pode ocorrer importantes alterações na mobilidade das cadeias poliméricas do hidrogel o que poderia refletir em variações na condutividade iônica do hidrogel. A aplicação dos hidrogéis de PAAm contendo PEDOT/PSS como capacitores foi investigada, com resultados bastante interessantes, recentemente publicados ${ }^{[14]}$.

\section{Conclusões}

Foi possível obter hidrogéis condutores através de polimerização química do monômero AAm em solução aquosa contendo o polímero condutor PEDOT/PSS e agente de reticulação MBAAm com diferentes formulações. Por meio de planejamento fatorial $2^{3}$ completo com ponto central foram quantificados os efeitos principais e de interação dos fatores: concentração de AAm e de MBAAm e quantidade de

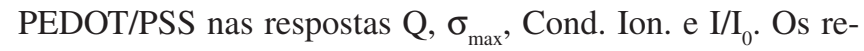
sultados dos estudos realizados por planejamento fatorial mostraram que a mudança na concentração de PEDOT/PSS (de $1,0 \%$ para $5,0 \%$, ou vice-versa) provoca alterações estatisticamente significativas ( $95 \%$ de confiança) somente na propriedade $\mathrm{I} / \mathrm{I}_{0}$. Por outro lado, as análises das respostas acima mencionadas, mostraram que a estabilidade mecânica dos hidrogéis é preservada mesmo após a adição de PEDOT/PSS e a presença do PEDOT/PSS aumenta o entrelaçamento na matriz de PAAm sem provocar mudanças nas propriedades hidrofílicas e mecânicas dos hidrogéis. A condutividade iônica do hidrogel, nos níveis de PEDOT/PSS estudados, está relacionada à quantidade de água presente no hidrogel (o que está indiretamente ligado à concentração de AAm e de MBAAm, e não à quantidade de cadeias de PEDOT/PSS presentes no interior do hidrogel). Dessa forma, torna-se possível obter hidrogéis com hidrofilicidade e transparência controladas, o que é muito importante para a aplicação desses materiais, principalmente como dispositivos ópticos.

\section{Agradecimentos}

Os autores agradecem à CAPES, CNPq, FAPESP pelo apoio financeiro.

\section{Referências Bibliográficas}

1. Routh, M. W.; Swartz, P. A \& Denton, M. B. - Anal. Chem., 49, p.1422 (1977).

2. Brereton; R. G. - Analyst, 112, p.1635 (1987).

3. Bruns, R. E.; Neto B. B. \& Scarmínio I. S. - "Como fazer experimentos". Editora da Unicamp, Campinas (2001).

4. Ismail, A. F. \& Lai, P. Y. - Sep. Purif. Technol., 40, p.191 (2004).

5. Boyac, I. H. - Biochem. Eng. J., 25, p.55 (2005).

6. Ahmad, A. L.; Ismail, S. \& Bhatia, S. - Environ. Sci. Technol., 39, p.2828 (2005).

7. Montgomery, D. C. - "Design and Analysis of Experiments", John-Wiley \& Sons, New York (1997).

8. Moura, M. R de., Guilherme, M. R., Campese, G. M., Radovanovic, E., Rubira, A. F. \& Muniz, E. C. - Eur. Polym. J., 41, p.2845 (2005).

9. Lin, J.; Tang, Q.; Wu, J.; \& Hao, S. - React. Funct. Polym., 67, p.275 (2007

10. Aouada, F. A.; Moura, M. R de; Fernandes, P. R. G.; Rubira, A. F. \& Muniz, E. C. - Europ. Polym. J., 41, p.2134 (2005).

11. Rai, P.; Majumdar, G. C.; DasGupta, S. \& De, S. - J. Food Eng., 64, p.397 (2004).

12. Girotto, E. M. \& Santos, I. A. - Química Nova, 25, p.639 (2002).

13. Muniz, E. C. \& Geuskens, G. - Macromolecules, 34, p.4480 (2001).

14. Aouada, F. A.; Guilherme, M. R.; Campese, G. M.; Girotto, E. M.; Rubira, A. F. \& Muniz, E. C. - Polym. Test., 25, p. 158 (2006).

Enviado: 09/07/07

Reenviado: 23/08/07

Aceito: $29 / 08 / 07$ 